\title{
El lenguaje del texto escolar de Ciencias Naturales: Problemas para el aprendizaje.
}

\section{The language of textbooks: Obstacles for learning.}

\section{Por: Chamorro Diana; Barletta Norma*}

Resumen: El análisis del discurso de varios textos escolares de Ciencias Naturales, en el ciclo de educación básica desde la perspectiva sistémico funcional, demuestra que el lenguaje de los textos puede presentar dificultades que requieren, por parte del docente, un manejo conciente con el fin de contribuir al logro de aprendizajes significativos.

Las investigaciones ${ }^{\dagger}$ permitieron identificar en los libros características discursivas tales como: incompletud de la información, referencias erróneas o sin antecedentes, relaciones entre las partes del texto, nominalizaciones, y ambigüedades en el lenguaje que al no estar adecuadamente mediadas por el docente, se constituyen en una dificultad para el aprendizaje Por lo tanto, consideramos necesario subrayar un trabajo conjunto entre investigadores del lenguajey $1 @$ s maestr@s de ciencias a fin de que se tome conciencia del lenguaje presente en los textos escolares en el aula, y las formas de tratarlo para el logro de aprendizajes significativos.

Palabras clave: texto escolar, análisis del discurso, aprendizaje.

Abstract: The analysis of the discourse of several Natural Science textbooks from the systemic functional perspective, as well as ethnographic studies carried out in junior high school classrooms have demonstrated that the language of textbooks can pose students a number of difficulties which require the teacher to develop a certain degree of awareness in order to be able to contribute to his/her students' meaningful learning.

The studies allowed to identify a number of discourse features that may cause problems for the students to understand the information. The features discussed here are incompleteness of information, wrong references or references without

Fundación Universidad del Norte Grupo de Investigación: Lenguaje y Educación.dchamorro@uninorte.edu.co, nbarlett@uninorte.edu.co

+ El Colectivo Urdimbre ha realizado investigaciones sobre el lenguaje de los textos escolares en 10 contextos de aula de básica secundaria. 
clear antecedents, relations among the different parts of the text, nominalizations, and ambiguities in the language. These characteristics need to be mediated by the teachers to help students learn. Thus, it is necessary to highlight the need for joint work between language researchers and the teachers of Natural Science, in order to develop awareness of the difficulties posed by the language of textbook and to be able to use it as a mediation to facilitate higher levels of learning.

Key words: School textbook, discourse analysis, learning

\section{INTRODUCCIÓN}

Actualmente, en los procesos de enseñanza-aprendizaje en las aulas de las escuelas secundarias del sector público, una de las mediaciones más importantes para la transmisión y/o construcción de conocimientos es el texto escrito, generalmente en la forma de libros de texto escolar. Es por esta razón que el Colectivo Urdimbre*, del Grupo de Investigación Lenguaje y Educación de la Universidad del Norte, se ha dedicado desde el año 1993 al estudio del lenguaje de los libros escolares y a su relación con los procesos de aprendizaje, la ideología y la formación de ciudadanas y ciudadanos. Hasta el momento, nuestras investigaciones se han centrado en Ciencias Naturales y Ciencias Sociales, mediante una metodología en la que combinamos el estudio etnográfico de la interacción maestr@-textoalumn@en el aula y el análisis sistémico funcional del discurso de los escritos. Los resultados nos han permitido establecer una categorización del aprendizaje en relación con las dificultades inherentes en el lenguaje de los textos escolares y las debilidades de la mediación realizada por los/as docentes. Los/as estudiantes observados/as tienden a mostrar evidencia de niveles muy limitados de aprendizaje (Barletta y Moss 1999). A lo largo de nuestros análisis de observaciones de interacciones en el aula y de entrevistas con estudiantes, hemos identificado seis niveles de aprendizaje: Enunciado no pertinente, Recitado mecánico, Elaboración incorrecta, Comprensión académica, Interacción entre significados académicos y experienciales, Transformación de significados de la experiencia.

El propósito de este artículo es dar a conocer algunas de las características del lenguaje de dos secciones (la respiración y estudiemos los fluidos) de un libro de texto de Ciencias Naturales de octavo grado de la editorial Voluntad que se constituyen en obstáculo para el

* Este artículo es producto de las investigaciones "El Lenguaje de los textos escolares en las áreas de Ciencias Sociales y Ciencias Naturales y su influencia en los procesos de aprendizaje" Fase I y Fase II; "Implementación de una propuesta metodológica para el manejo de los textos escolares y análisis de sus resultados" y "La relación entre lenguaje e ideología en la interacción maestr@-texto-alumn@ en las clases de Ciencias Naturales y su influencia en el proceso de formación de ciudadanas y ciudadanos" realizada por el Colectivo Urdimbre bajo los auspicios de la Colciencias y la Dirección de Investigaciones y Proyectos de la Universidad del Norte. En Ciencias Naturales en total se han observado 6 contextos de aula en los cuales se han utilizado 6 libros de texto de las editoriales: Santillana, Voluntad y Norma. Estos textos fueron escogidos en su momento después de indagar mediante encuesta acerca de los textos más usados en grado 8 . 
aprendizaje. Justificaremos y explicaremos uno a uno los análisis de varios de los aspectos identificados. Finalmente, haremos algunas recomendaciones sobre el tratamiento de dichas características.

\section{Incompletud de la información}

En los textos escolares podemos distinguir varios tipos de texto de acuerdo con la clase de información que presentan (Davies, 1992; Davies y Greene, 1984): Proceso Biológico, Ley o Principio Conducta, Estructura Organizacional, Estructura Física, Clasificación, entre otros. De esta manera, según la temática, es posible identificar unos constituyentes comunes a los textos de un mismo tipo. Algunos de estos constituyentes informativos se podrían considerar obligatorios para facilitar la comprensión de fenómeno, por ejemplo: un pasaje que alude a estructura física resultaría poco claro si, aunque se describieran las partes de que está compuesta una entidad, no se dijera la función de cada una de ellas. Los resultados de nuestras investigaciones sugieren que la ausencia de información sobre la función de las partes dificulta a los lectores encontrarle sentido a la existencia de las partes, establecer relaciones entre ellas y reconocerlas como integrantes de procesos vitales, por lo tanto, serían más difíciles de recordar. Estos procesos se hacen más evidentes cuando no hay un acompañamiento efectivo por parte del docente.

Nuestras investigaciones, en el contexto de Ciencias Naturales, nos han permitido corroborar la presencia de los tipos de texto ya identificados por Davies y Greene. En este artículo, haremos referencia a Procesos Biológicos y Ley o Principio.

Proceso Biológico. Esta temática o tópico comprende las unidades informativas que aluden a la descripción de funciones orgánicas y otros procesos de tipo biológico mediante los siguientes constituyentes: objeto/material/participante, localización, acción, instrumento, propiedad, tiempo/etapa, condición y efectos (Colectivo Urdimbre, 2000). Es decir, este tópico describe cómo una entidad bajo unas condiciones, realiza unas funciones y/o acciones propiciadas por algunos elementos en ciertos espacios y momentos.

El conocimiento de la forma como se organizan los textos y como se distribuye su información, permite no solo la comprensión sino evaluar su completud y ser conscientes de que los procesos en nuestro organismo ocurren como respuesta a ciertos tipos de estímulos $\mathrm{y}$, asimismo, desencadenan efectos en nosotros, en otros organismos o en el medio ambiente.

En un pasaje de un libro de Ciencias Naturales $\ddagger$ grado 8 en el que se describen las características del proceso de respiración cutánea, hicimos el ejercicio de identificar los componentes de acuerdo con lo que esperamos tenga un texto de este tipo (Tópico Proceso Biológico). Veamos cómo reza el pasaje.

\$ Inteligencia Científica 8. Editorial Voluntad 


\begin{abstract}
[1] Respiración cutánea
Consiste en la difusión del oxígeno a través de la piel o tegumento hasta los vasos sanguíneos y la eliminación del gas carbónico desde los vasos sanguíneos por la piel al medio. La sangre transporta el oxígeno y a nivel celular realiza un nuevo intercambio de gases respiratorios por difusión; el oxígeno combuste las sustancias protoplasmáticas y libera la energía (IC. 70:5).
\end{abstract}

El análisis de los constituyentes de este pasaje distribuidos en una tabla, se ilustra así:

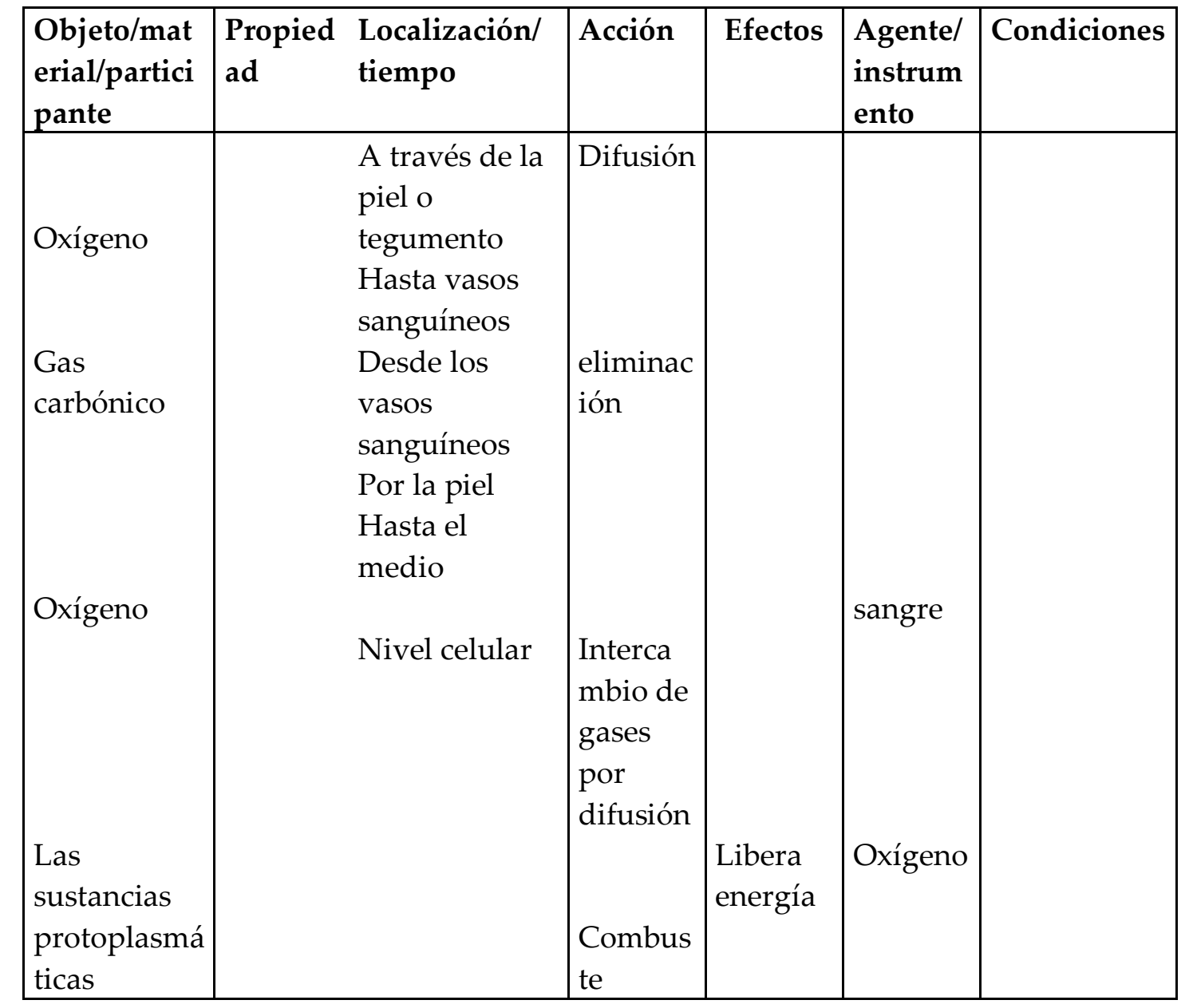

Como podemos apreciar, en el fragmento se deja de decir qué hace que el oxígeno entre a través de la piel, es decir, no se especifican las condiciones. Nótese que no se enuncian tampoco las propiedades que debe tener la piel para que ese intercambio pueda realizarse.

Por otro lado, cabe anotar que la forma de expresar la localización "la eliminación del gas carbónico desde los vasos sanguíneos por la piel al medio" puede llevar al estudiante a confundir el agente por el lugar ya que el uso de la preposición "por" resulta ambiguo. Si leemos el 
texto desprevenidamente, podríamos suponer incluso que el oxígeno entra indefinidamente al cuerpo. El uso de la preposición "por" parece indicar que el proceso de eliminación del gas carbónico no está en relación con el oxígeno, más bien sugiere que la piel es el agente que obliga al gas carbónico a salir.

Otro de los tópicos identificados en el mismo texto es de de Tópico de Ley o Principio. Este se caracteriza por describir una regla explicativa general con ejemplos de su aplicación e implicaciones (Lunzer et al 1984). En sus análisis, Davies identifica los constituyentes: ley o principio, condición, ejemplos, medidas o pruebas y aplicación (Moss et al, 2003: 33). No obstante, los libros no siempre suministran esta información. Veamos un ejemplo:

\section{[2] Principio de Pascal}

Las fuerzas pueden actuar fácilmente sobre los sólidos cuando se aplican sobre un punto o área muy pequeña, produciendo el efecto deseado.

En los líquidos no se puede proceder de la misma forma, ya que es necesario que se encuentre encerrado y la fuerza se ejerza mediante una superficie. Es imposible desplazar una masa de gas o líquido sin disponer de una superficie sólida que transmita al líquido o al gas la fuerza aplicada.

Este hecho fue descubierto por Pascal quien formuló el principio que lleva su nombre:

"Al ejercer una presión adicional sobre un fluido esta presión se transmite por igual a todos los puntos del fluido y a las paredes del recipiente que lo contiene". (IC. 149 -150.1)

He aquí el análisis detallado de los constituyentes:

\begin{tabular}{|l|l|l|l|l|l|}
\hline $\begin{array}{l}\text { Párra } \\
f o\end{array}$ & \multicolumn{5}{|c|}{ Constituyentes informativos } \\
\hline & Ley o Principio & Condiciones & $\begin{array}{l}\text { Instanc } \\
\text { ias }\end{array}$ & $\begin{array}{l}\text { Medid } \\
\text { as/ } \\
\text { Prueba } \\
\text { s }\end{array}$ & $\begin{array}{l}\text { Aplicacio } \\
\text { nes }\end{array}$ \\
\hline 149. & $\begin{array}{l}\text { Principio de Pascal. Las } \\
\text { fuerzas pueden actuar } \\
\text { fácilmente sobre los sólidos }\end{array}$ & $\begin{array}{l}\text { Cuando se aplican } \\
\text { sobre un punto o } \\
\text { área muy pequeña } \\
\text { produciendo el } \\
\text { efecto deseado. }\end{array}$ & $\begin{array}{l}\text { Ya que es necesario } \\
\text { que se encuentre } \\
\text { encerrado y la } \\
\text { fuerza se ejerza } \\
\text { mediante una } \\
\text { superficie. }\end{array}$ & & \\
\hline $\begin{array}{l}149 . \\
8\end{array}$ & $\begin{array}{l}\text { En los líquidos no se puede } \\
\text { actuar de la misma forma }\end{array}$ & & \\
\hline
\end{tabular}




\begin{tabular}{|l|l|l|l|l|}
\hline & $\begin{array}{l}\text { Es imposible desplazar una } \\
\text { masa de gas o líquido }\end{array}$ & $\begin{array}{l}\text { Sin disponer de } \\
\text { una superficie } \\
\text { sólida que } \\
\text { transmita al } \\
\text { líquido o al gas la } \\
\text { fuerza aplicada. }\end{array}$ & & \\
\hline 150. & $\begin{array}{l}\text { Al ejercer una presión } \\
\text { adicional sobre un fluido, } \\
\text { esta presión se transmite } \\
\text { por igual a todos los puntos } \\
\text { del fluido y a las paredes } \\
\text { del recipiente que lo } \\
\text { contiene. }\end{array}$ & & & \\
\hline
\end{tabular}

Obsérvese que falta información relacionada con: instancias, medidas/pruebas y aplicaciones. Al parecer, los autores del texto consideran que esta información no es necesaria para la comprensión del principio explicado.

La falta de información no es la única dificultad que hemos identificado con respecto al desarrollo de los tópicos. Otro aspecto que resulta complejo para la comprensión de la información estriba en que en algunos casos, se superponen los tipos de tópico en diferentes niveles de generalidad, de manera que un tópico de menor nivel de generalidad puede entrar a formar un constituyente de otro tópico de mayor generalidad. En el texto de Ciencias Naturales al que nos referimos aquí (ver apéndice 1), hemos identificado tres niveles de generalidad: el tópico estructurador de Definición se presenta como el más general, o macro-tópico; en segundo nivel, encontramos el tópico de Clasificación, y finalmente, en tercer nivel, el más específico, se ubica el tópico de Proceso Biológico que analizamos anteriormente, y que a su vez, aparece como constituyente "clase" del tópico Clasificación.

El texto comienza definiendo el fenómeno "respiración", luego especificando la clase a la que pertenece, "es un proceso", y luego indicando características distintivas (definición por clase y características): "mediante el cual los seres vivos liberan la energía almacenada en las sustancias protoplasmáticas".

Luego, el texto presenta diversos tipos de clasificación de la respiración sin establecer claramente cuál es el criterio seguido para la misma. Cabe resaltar que los tópicos de clasificación cumplen una doble función: informar y estructurar la información. Normalmente los constituyentes son: clase, rasgos distintivos, condición, función y ejemplos. Pero además, es esencial que se explicite el criterio seguido para establecer la clasificación. Esto resulta especialmente importante para un texto que como éste, presenta varios tipos de clasificación anidadas. 
Esta forma de estructurar la información obliga a que el estudiante se enfrente al menos a dos retos: 1. superar la falta de información con respecto al proceso de respiración cutánea: 2 . reconstruir la conexión con el esquema macro que plantea el texto:

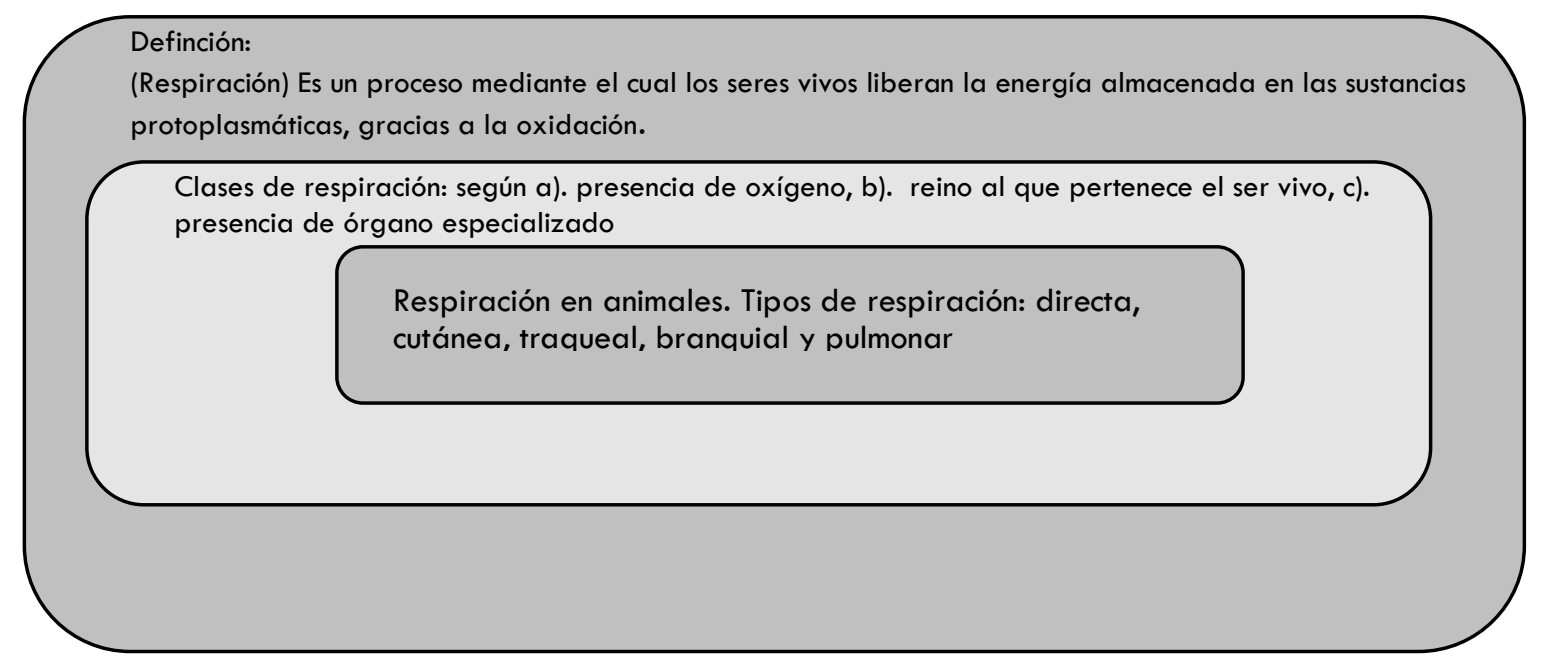

La claridad del criterio para establecer la clasificación y la explicitación adecuada del mismo en la parte introductoria de los tópicos (Lunzer et al 1994), es fundamental para limitar el campo de estudio, ya que permite reconstruir la estructura del texto, entender las relaciones de las partes entre sí. Asimismo, sirve de orientación del lector hacia los aspectos más importantes a tratar, sirviendo así como organizador del texto (función textual), lo cual contribuye notablemente a la cohesión del texto, y, en consecuencia, al procesamiento más ágil de la información por parte del estudiante.

Los resultados de nuestras investigaciones indican que también hay problemas con las formas en que se relacionan las oraciones al interior del texto: cohesión (Halliday y Hasan, 1976)

\section{Referencias erróneas o sin antecedentes}

La cohesión se logra en muchas ocasiones mediante el uso de las palabras y recursos gramaticales que posibiliten la reconstrucción por parte del lector de estas relaciones. Volvamos al ejemplo dos y analicemos en él la cohesión. Nótese que la primera oración del párrafo citado inicia con "Las fuerzas". El uso del artículo definido "Las" (en la oración "las fuerzas pueden actuar fácilmente sobre los sólidos cuando se aplican sobre un punto o área muy pequeña, produciendo el efecto deseado") parece aludir a algunas fuerzas antes mencionadas o presumir que los lectores saben a cuáles fuerzas se está refiriendo el texto. Sin embargo, anteriormente no ha habido mención de fuerzas y no es posible saber quién aplica las fuerzas, ni cuándo, ni por qué ni para qué, puesto que sólo se menciona un "efecto deseado". 
En la siguiente cláusula, "En los líquidos no se puede proceder de la misma forma", los lectores deben decidir que "proceder de la misma forma" se refiere a "aplica[r] fuerzas sobre un punto o área muy pequeña, produciendo el efecto deseado", una deducción exigente para el lector inexperto, especialmente porque no se ha establecido cuál es el efecto deseado. Y si miramos la oración completa: "En los líquidos no se puede proceder de la misma forma, ya que es necesario que se encuentre encerrado", vemos que no hay claridad con respecto a quién o qué debe encontrarse "encerrado" ya que el sujeto de la oración es "los líquidos", pero estos están en plural y "encerrado" está en singular.

Veamos ahora cómo continúa el texto:

[3] Este hecho fue descubierto por Pascal quien formuló el principio que lleva su nombre.

“Al ejercer una presión adicional sobre un fluido esta

presión se transmite por igual a todos los puntos del fluido y a las paredes del recipiente que lo contiene".

Aquí es posible que los lectores se pregunten cuál es la presión "inicial" que se ejerce, ya que el principio de Pascal se refiere a una presión "adicional". Como se ve, el texto no facilita la recuperación de las relaciones entre cláusulas.

En otra sección titulada "Presión en un líquido", encontramos:

\section{[3] Presión en un líquido}

Cuando una persona se sumerge en una piscina soporta una presión ejercida por el agua. Esto se percibe especialmente en los oídos.

Mientras más profundo se encuentre el cuerpo, el líquido le ejerce mayor peso. Esto nos lleva a concluir que a mayor profundidad mayor presión. Este fenómeno se produce en todos los fluidos y se denomina presión hidrostática.

La presión hidrostática también depende de la densidad del líquido: a mayor densidad mayor presión (IC: 149)

Obsérvese que en el texto se afirma que "a mayor profundidad mayor presión" y "este fenómeno se denomina presión hidrostática". Asignarle el nombre de presión hidrostática a esta variable de la presión es incorrecto y esto es corroborado en la oración inmediatamente después: "La presión hidrostática también depende de la densidad del líquido: a mayor densidad mayor presión". El uso de "también" indica una relación de adición y semejanza, es decir, en ningún caso se ha definido la presión; se han explicitado dos variables de la presión hidrostática: profundidad del cuerpo y densidad del líquido. Sin embargo, es difícil que un lector inexperto de octavo grado reconstruya estas dos como tales. En el texto no se explica por qué razón se resalta la segunda variable (densidad) por medio del tipo y tamaño de letra, color e interlineado especiales. 


\title{
Relaciones entre las partes del texto
}

La cohesión de un texto debe ser reconstruida por los lectores. Esto se hace mediante las señales que ofrece un texto a nivel micro, pero también utilizando los esquemas de diagramación, organización y relaciones semánticas que los lectores han construido con base en su experiencia lectora.

Veamos cómo el texto en mención organiza la información que se va a presentar alrededor de los fluidos. El siguiente es un esquema de la información basado en el tamaño de las letras, su ubicación y su color.

\author{
[4] Los fluidos (fucsia) \\ Propiedades de los líquidos (azul) \\ La forma (rojo) \\ La densidad (rojo) \\ La presión (rojo) \\ Unidad de fuerza (azul) \\ Presión en un líquido (azul) \\ La presión hidrostática también depende de la densidad \\ del líquido: a mayor densidad mayor presión. (verde a doble espacio) \\ Características de la presión hidrostática (rojo) \\ Principio de Pascal (azul) \\ "Al ejercer una presión adicional sobre un fluido, esta presión \\ se transmite por igual a todos los puntos del fluido y a las paredes \\ del recipiente que lo contiene" (fucsia a doble espacio)
}

En esta diagramación es difícil reconocer la relación entre las subsecciones tituladas "Unidad de fuerza", "Presión en un líquido" , y "Principio de Pascal", puesto que estos temas están desarrollados como separados y no se sabe cuál es el principio de organización de la información, su jerarquización o la relación que conecta lo uno con lo otro. Por el contrario, es aparentemente sencillo reconocer que "la forma", "la densidad", y "la presión" son propiedades de los líquidos. Hay que anotar, sin embargo, que la información que se presenta bajo el subtítulo "La densidad" se refiere inicialmente a la noción de densidad en general y luego a la densidad del cobre, para luego compararla con la del alcohol. Bajo el subtítulo "La presión" (como propiedad de los líquidos), la información nunca se refiere a líquidos específicamente, sino que se da el ejemplo de un par de libros que están sobre una superficie sólida.

Cuando finalmente entran a colación los líquidos, se trata de una nueva sección titulada "La presión en un líquido", y en este apartado, se habla de la presión ejercida por el agua a los cuerpos sumergidos en ella y que depende de la profundidad a la que se encuentre el cuerpo y de la densidad del líquido. Aquí pareciera que nada de lo anterior fuera relevante, pues no se retoma el concepto de fuerza, el área, ni la unidad de fuerza. 
Esta manera de organizar la información en un texto se asemeja a la de los textos tipo colonia (Hoey, 2001), como los diccionarios, las enciclopedias, los avisos clasificados, el horóscopo, los cuales no presuponen una lectura lineal, sino una lectura selectiva de secciones, de acuerdo con la información específica que se esté buscando. En estos textos, la lectura de la sección o las secciones previas no son imprescindibles para la comprensión de secciones posteriores, pues cada una de ellas es bastante independiente.

Por supuesto, para poder hacer una lectura efectiva de este tipo de texto, el lector debe estar familiarizado con el principio organizativo del texto. En el caso del texto bajo análisis, este principio no está claro. Posiblemente se aborde linealmente (como generalmente se hace con los textos escolares) con la esperanza de que los estudiantes reconstruyan el tema en su conjunto y establezcan las relaciones que, desafortunadamente, están ausentes.

Hay otros aspectos que complejizan aún más la comprensión de este texto, veamos:

\section{Nominalización}

La nominalización como un tipo de metáfora gramatical, constituye una característica esencial del discurso científico (Halliday 2004b). Es la expresión de procesos y propiedades en forma de sustantivos, en vez de verbos y adjetivos. A nivel conceptual, la nominalización "mantiene inmóvil a la realidad para permitir la observación y experimentación" (Halliday y Martin, 1993: 15). Los procesos y las propiedades se objetivizan, y se representa un mundo en el que los objetos predominan y los procesos sirven simplemente para definir y clasificar.

En el caso de los textos de Ciencias Naturales el peso de la nominalización cae sobre los procesos fisiológicos, que no se presentan como procesos dinámicos en estado de constante cambio e interacción con el medio circundante, sino como objetos fijos, sin vida, de alguna manera, independientes de los seres que los realizan. Ejemplo de esto es el poco uso del verbo respirar el cual exige un sujeto viviente. En el texto escolar que nos ocupa, la objetivización del proceso fisiológico "respirar" se inicia con la nominalización "respiración" y se completa mediante el uso del verbo "tener" y "presentar" que sugieren más bien una relación entre dueño y objeto externo que entre un ser y sus procesos vitales:

\footnotetext{
“Los animales tienen respiración aerobia" (IC, 70:2)

"Los protistos como la euglena y el paramecio tienen respiración aerobia" "Los hongos presentan respiración anaerobia" (IC: 69:1)
}

Otra dificultad que presenta el discurso altamente nominalizado en comparación con el discurso congruente, es la pérdida de información acerca de tiempo, transitividad y modalidad. Podemos encontrar ejemplos de este problema en el siguiente pasaje: 


\section{[5] Respiración en plantas}

Las plantas necesitan energía para cumplir sus funciones vitales; esa energía la suministra la respiración.

Intercambio gaseoso: las plantas inferiores y algunas acuáticas lo hacen por difusión, las superiores poseen órganos especializados localizados en diferentes partes de la planta. Estos son: estomas, lenticelas y neumatóforos. (IC; 69)

Las nominalizaciones subrayadas dejan sin responder las siguientes preguntas: ¿Quién realiza el proceso de respirar? ¿Cómo se realiza? ¿Qué se difunde? ¿Quién difunde? ¿Bajo qué circunstancias se difunde?

Otro ejemplo de cómo como las metáforas gramaticales suelen opacar el sentido de un texto, se puede apreciar en el siguiente ejemplo en el que se han subrayado las nominalizaciones:

Las fuerzas pueden actuar fácilmente sobre los sólidos cuando se aplican sobre un punto o área muy pequeña, produciendo el efecto deseado. (IC: 149.7)

En el ejemplo, podemos apreciar que las fuerzas actúan por sí mismas, no se explicita un agente que ejerza la fuerza y ante la ausencia de un referente anterior, a pesar del artículo definido, puede resultar una proposición demasiado abstracta, difícil de comprender para un lector inexperto de octavo grado.

Esta ausencia de agente se acentúa mucho más cuando afirman "se aplican sobre un punto o área muy pequeña”, cabe aquí preguntarse ¿cuál es la fuente de la fuerza? ¿de dónde surgen las fuerzas? ¿bajo qué circunstancias? Es posible que el lector pueda inferir que las fuerzas actúan por sí mismas. Por su parte, "el efecto deseado" carece de un referente reconocible ¿a qué efecto se refiere? ¿quién desea provocar determinado efecto? Esta falta de agencialidad, característica de la nominalización, subraya el carácter abstracto de la ciencia dado a este pasaje del texto.

La nominalización es más difícil de comprender cuando se acompaña de una metáfora léxica. Veamos:

\section{La respiración}

Es un proceso mediante el cual los seres vivos liberan la energía almacenada en las sustancias protoplasmáticas, gracias a la oxidación. (IC, 68)

Aquí estamos ante la presencia de dos tipos de metáforas: léxica (liberan la energía) y gramatical (oxidación). Cuando el texto dice que los seres vivos liberan energía, sugiere que la energía está guardada como lo sugiere la palabra "almacenada" y que 
luego sale gracias a un proceso denominado "oxidación". Este por su naturaleza metafórica no aclara al estudiante quién es el agente, en qué consiste el oxidarse, por qué razón o circunstancias ocurre. Ante la falta de información que implica la metáfora gramatical, y utilizando su conocimiento del sentido cotidiano, literal, del verbo "oxidar", los estudiantes posiblemente confundan la oxidación a nivel celular con aquélla que tiene lugar en los metales como el hierro en lugares con alta humedad.

En los ejemplos dados, los conceptos, objetivizados y empacados, es decir, inmovilizados y desligados de agentes y tiempo concreto, se presentan al lector sin ninguna explicación acerca de su origen ni de su relación con el contexto ni con el mundo externo. En estas circunstancias las posibilidades de interacción significativa con el texto se reducen al mínimo y al lector no poseedor de los conocimientos previos que le permitirían desempacar la metáfora sin referencia al texto, no le queda otra alternativa sino aceptar y memorizar pasivamente o simplemente rechazar un discurso que le resulta enajenante (lo que F. Davies denomina "lectura rechazante" (rejective reading), comunicación personal 1992).

\section{Ambigüedades en el lenguaje}

Una parte de la ambigüedad se da por el uso de terminología inconsistente:

$$
\begin{aligned}
& \text { "[... ] la unidad de fuerza es el newton..." (IC: 149. 2) } \\
& \text { “ [... ] un automóvil que pesa } 38000 \text { Newtons... F2 = } 38000 \text { newton..." (IC: } \\
& 150.5)
\end{aligned}
$$

Aquí, la relación o la diferencia entre peso y fuerza siguen siendo confusas, a pesar de que son conceptos centrales del capítulo.

La presión: el peso del libro que actúa sobre la superficie de menor área ejerce mayor presión. La presión depende de dos factores: la fuerza aplicada y la superficie sobre la que actúa. (IC. 148. 8)

A pesar de que en el texto hay una ilustración de dos libros sobre una superficie rectangular (Ver apéndice 2), en el citado pasaje no se hace alusión directa al mismo. También se afirma que la presión depende de dos factores: la fuerza aplicada y la superficie sobre la que actúa; pero, no se dice que la fuerza es igual al peso y que a mayor fuerza, mayor presión y, por el contrario, a menor superficie, mayor presión. La reconstrucción de la forma invertida como funcionan estos dos factores, se deja de tarea al estudiante.

\section{La interacción en el aula}

Nuestras investigaciones nos sugieren que hay poca conciencia de parte de los docentes con respecto a las características lingüísticas de los textos. Esto tal vez puede estar asociado con 
la confianza depositada en los libros de texto, la creencia de que como estos reflejan los contenidos basados en la lógica formal de la disciplina, no crean dificultades en el proceso de aprendizaje de los alumnos (Porlán, 1997).

La conciencia del lenguaje con el que se presenta la información en los textos escolares, daría al docente los elementos para realizar una revisión previa de las posibles dificultades, los vacíos, ambigüedades o la complejidad de los mismos y lo llevaría a incluir dentro de la preparación de sus clases, actividades, talleres, preguntas que apunten a recuperar información previa, identificar nominalizaciones para explicitar los posibles agentes, tiempos y condiciones en que se realizan los procesos, establecer relaciones, aclarar la lógica y organización del texto. Es decir, prepararía a los estudiantes a interpretar a partir de los textos.

Lo que hemos observado, desafortunadamente, indica que en muchos casos el tratamiento que se hace del texto es superficial, parece que consistiera en avanzar por los capítulos, mas no en desarrollar la comprensión de los fenómenos naturales o en volverlos capaces de abordar nuevos textos en el futuro. Como consecuencia, hemos evidenciado interacciones poco significativas que dan lugar a un aprendizaje de tipo mecánico o a proposiciones que no corresponden con la realidad y que no son debidamente explicadas. A continuación, presentamos el extracto de una clase de Ciencias Naturales en la cual se está haciendo la "puesta en común" de un taller que consistía en varias preguntas dictadas por la maestra. Los estudiantes previamente han trabajado en grupos y han resuelto por escrito las preguntas dictadas por la maestra utilizando la información del libro de texto.

\begin{tabular}{|c|c|c|c|}
\hline 1 & A: & Profe, falta la densidad & \\
\hline 2 & P: & $\begin{array}{l}\text { Ah, seguimos con otra propiedad que } \\
\text { tienen los fluidos, especialmente los } \\
\text { líquidos. ¿Cuál es la propiedad? A ver, } \\
\text { G [...] }\end{array}$ & $\begin{array}{l}\text { Pregunta por } \\
\text { dato }\end{array}$ \\
\hline 3 & A: & $\begin{array}{l}\text { Es el volumen que contienen las } \\
\text { diferentes sustancias que existen en la } \\
\text { naturaleza. Ejemplo: La masa de un } \\
\text { centímetro cúbico de cobre es } 8.9 \\
\text { gramos mientras que el mismo volumen } \\
\text { de alcohol tiene una masa de } 0.81 \\
\text { gramos. }[\ldots]\end{array}$ & $\begin{array}{l}\text { No } \\
\text { correspondencia }\end{array}$ \\
\hline 4 & A: & $\begin{array}{l}\text { Las diferentes sustancias que existen en } \\
\text { la naturaleza se caracterizan porque } \\
\text { para un mismo volumen tienen } \\
\text { diferente masa. Así, por ejemplo, la } \\
\text { masa de un centímetro cúbico de cobre } \\
\text { es } 8.9 \text { gramos, mientras que el mismo }\end{array}$ & $\begin{array}{l}\text { Recitación } \\
\text { discurso texto }\end{array}$ \\
\hline
\end{tabular}




\begin{tabular}{|c|c|c|c|}
\hline & & $\begin{array}{l}\text { volumen de alcohol, tiene una masa de } \\
0,81 \text { gramos. }\end{array}$ & \\
\hline 5 & P: & Densidad... repite densidad & \\
\hline 6 & A: & $\begin{array}{l}\text { Las diferentes sustancias que existen en } \\
\text { la naturaleza se caracterizan porque } \\
\text { para un mismo volumen tienen } \\
\text { diferente masa. Así, por ejemplo, la } \\
\text { masa de un centímetro cúbico de cobre } \\
\text { es } 8.9 \text { gramos, mientras que el mismo } \\
\text { volumen de alcohol, tiene una masa de } \\
0,81 \text { gramos. } \\
\text { La densidad de una masa por unidad de } \\
\text { volumen de dicha sustancia. }\end{array}$ & $\begin{array}{l}\text { Recitación } \\
\text { discurso texto }\end{array}$ \\
\hline 7 & P: & $\begin{array}{l}\text { O sea, que entonces densidad es igual a } \\
\text { ¿qué? }\end{array}$ & $\begin{array}{l}\text { Pregunta por } \\
\text { dato }\end{array}$ \\
\hline 8 & A: & Masa & $\begin{array}{l}\text { No } \\
\text { correspondencia }\end{array}$ \\
\hline & & {$[\ldots]$} & \\
\hline 9 & As: & Ahora sí seguimos, pues & \\
\hline $\begin{array}{l}1 \\
0\end{array}$ & A: & $\begin{array}{l}\text { La presión, seño. El peso del libro que } \\
\text { actúa sobre la superficie de menor área } \\
\text { ejerce mayor presión. La presión } \\
\text { depende de dos factores. La fuerza } \\
\text { aplicada y la superficie sobre la que } \\
\text { actúa. }\end{array}$ & $\begin{array}{l}\text { Recitación } \\
\text { discurso texto }\end{array}$ \\
\hline $\begin{array}{l}1 \\
1\end{array}$ & P: & $\begin{array}{l}\text { Sobre la que actúa...Entonces presión va } \\
\text { a ser igual,... ¿a qué? a la fuerza sobre } \\
\text { área. La presión va a ser igual a la } \\
\text { fuerza que actúa sobre cualquier objeto } \\
\text { pero teniendo en cuenta el área. } \\
\text { Entonces, la presión es igual a fuerza } \\
\text { sobre área. La pregunta siguiente... }\end{array}$ & $\begin{array}{l}\text { Evaluación } \\
\text { positiva } \\
\text { Definición }\end{array}$ \\
\hline
\end{tabular}

Como vemos en los turnos 3 y 4, las respuestas de los estudiantes contienen inexactitudes, como por ejemplo, que la densidad es "el volumen que contienen las sustancias", o que densidad es lo mismo que la masa. Si miramos el texto (Apéndice 2), la densidad no se define inmediatamente al introducir el término, aspecto que el alumno no parece haber detectado. Obsérvese que la maestra no se detiene a indagar sobre el origen de la confusión, y se limita a dar el turno a otro alumno, quien lee una definición tomada exactamente del libro. Más adelante la definición de densidad que se suministra dice textualmente: "La densidad de una sustancia es la masa por la unidad de volumen de dicha sustancia". A juzgar por la respuesta en el turno 4, el estudiante no está en capacidad de interpretar la 
frase "por la unidad de volumen de dicha sustancia" o no comprende su importancia y la maestra tampoco se detiene a explicarlo.

En el turno 11 la maestra escucha una respuesta tomada exactamente del texto, y procede a hacer una paráfrasis muy rápida de la información, con preguntas retóricas que ella misma se responde. Es decir, no existe ninguna evidencia de que los estudiantes hubieran producido discursos que demostraran una reformulación correcta de los conceptos en sus propias palabras o de que la maestra los hubiera orientado en ese proceso.

\section{CONCLUSIÓN}

En la interacción que reproducimos anteriormente se destaca la ausencia de apoyo de la maestra para solucionar problemas de comprensión de los alumnos y la aceptación de la repetición del lenguaje del texto como conductas válidas. La maestra no se percata de la naturaleza de las dificultades de los alumnos y éstos posiblemente no se percatan de la naturaleza de su equivocación o de que es posible llegar a otros tipos de aprendizaje.

Nuestros estudios señalan que la incompletud de la información, referencias erróneas o sin antecedentes, relaciones poco claras entre las partes del escrito, nominalizaciones, y ambigüedades en el lenguaje, sumado a la poca conciencia del maestro/a de la presencia de éstas, conducen a que los/as estudiantes alcancen sólo dos niveles de aprendizaje (Colectivo Urdimbre, 2000): recitado mecánico o elaboración incorrecta de la información que allí se presenta.

Para que haya un trabajo con el libro escolar que apunte a niveles mayores de aprendizaje es necesario evaluar aspectos del texto como: de qué tipo de temática se trata, o, en términos de Davies, qué tipos de tópico se están desarrollando, y esto implica determinar qué tipo de constituyentes informativos se esperaría encontrar, cuáles están desarrollados, y si hace falta alguno que sea importante para la comprensión del fenómeno; igualmente, es necesario hacer un análisis de la presentación y organización general del pasaje a estudiar, determinar, por ejemplo, si se está describiendo una clasificación o una taxonomía, si está claro cuál es la entidad o fenómeno cuyas clases se están describiendo, así como el criterio de la clasificación; qué tanto la diagramación del texto contribuye a aclarar la relación entre las diferentes partes.

Por otro lado, es esencial identificar las nominalizaciones presentes en el escrito para evaluar si en el texto mismo, o en los conocimientos previos de los estudiantes, es posible rescatar la información que se pierde cada vez que estamos ante este tipo de metáfora, como por ejemplo el agente del proceso, las condiciones, el tiempo en que se realiza. Pueden ser particularmente difíciles aquellos pasajes que contienen nominalizaciones de procesos que no han sido explicados, o los que contienen varias nominalizaciones seguidas. 
Igualmente, objeto de análisis debe ser el vocabulario, no solamente los términos específicos que se introducen en un pasaje, sino también de los que se supone que ya se estudiaron, así como aquellas palabras, que pueden tener acepciones en el lenguaje cotidiano y que pueden crear confusión.

El objetivo de estos análisis por parte de los docentes debería ser, en caso de identificar características potencialmente problemáticas, estar en mejor posición para hacer indagaciones más específicas sobre la comprensión de los estudiantes, para suministrar información faltante, señalar la lógica del texto, explicitar las relaciones entre las partes, explicitar la información que está dicha de manera muy sintética mediante las nominalizaciones, parafrasear las oraciones. Asimismo, serviría para preparar actividades de comprensión que hagan que el estudiante utilice sus propias palabras y estructuras para decir lo mismo que el libro, que le hagan caer en cuenta de la estructura de la información, y la forma como el texto mismo ayuda (o no) al lector a orientarse dentro de él mismo, actividades que les ayude a identificar los vacíos en la misma para que no mitificar el texto escolar y lo cuestionen cada vez que no comprendan parte de él.

El estar más consciente del lenguaje en general, de las características discursivas de los libros escolares, permitiría al docente estar alerta a los enunciados emitidos por los estudiantes que son tomados literalmente del texto y, por supuesto, de las distorsiones con respecto a la información que trae el escrito, y también de los enunciados que, siguiendo al texto, son confusos o no corresponden con lo que se sabe del mundo natural. Las interacciones que se generarían de docentes dispuestos a escuchar los enunciados de los alumnos podrían entonces ser menos controladas, más a modo de conversación y de construcción conjunta del conocimiento.

Nos parece que el trabajo interdisciplinario entre analistas del lenguaje y expertos en la disciplina podría ser muy provechoso para potenciar el texto escolar como mediación en el aula.

\section{Bibliografía}

- BARLETTA, N. y MOSS, G. (1999). Las palabras de los estudiantes como evidencia de aprendizaje. Lenguajes e Integración. En. Lenguajes e Integración. Memorias del II Encuentro Iberoamericano de Colectivos que Hacen Investigación en la Escuela. México: Universidad Pedagógica Nacional de México. 320p.

- COLETIVO URDIMBRE. 2000. Libros de texto y aprendizaje en la escuela. Sevilla: Diada . 96p.

- DAVIES, F. 1992. Introducing Reading. Harmondsworth: Penguin 116 p.

- DAVIES, F. and GREENE, I. 1984 Reading for learning in the sciences. Edinburgh: Oliver and Boyd. $144 \mathrm{p}$.

- HALLIDAY, M. A. K 2004. An Introduction to Functional Grammar. 3rd edition. Revised by C. M. I. M. Matthiessen. London: Arnold 689p. 
- HALLIDAY, M. A. K \& MARTIN, J. R. 1993. Writing science: Literacy and discursive power. London: Falmer 283 p.

- HALLIDAY, MAK and HASAN, R. 1976 Cohesion in English. London: Longman. 374p.

- HOEY, M. 2001.Textual interaction: An introduction to written discourse analysis. London: Routledge.203 p

- LUNZER, E.; GARDNER, K.; DAVIES, F.; GREENE, T. 1984. Learning from the written word. London: School Council Publication. 244 p.

- MOSS, G.; MIZUNO, J.; ÁVILA, D.; BARLETTA, N.; CARREÑO, S.; CHAMORRO, D. \& TAPIA, C. 2003. Urdimbre del texto escolar: ¿Por qué resultan difíciles algunos textos? 2a edición. Barranquilla: Ediciones Uninorte 129 p.

- PORLAN, R. 1997. Constructivismo y escuela. 4 Ed. Sevilla: Diada 194 p. 


\section{Apéndice 1}

\section{siny}

Estandar de contenido: Identifico la inportancia de la respinacion.

\section{La respiración}

Es un proceso mediante el cual los seres vivos liberan la energia almacenada en las sustancias protoplasmáticas, gracias a la oxidación.

La energia liberada en la respiración es utilizada por el ser vivo para cumplir las funciones vitales. Los seres vivos poco evolucionados no poseen órganos especializados para el intercarnbio de los gases; los más evolucionados presentan aparato respiratorio.

\section{Clases de respiración}

La respiración puede ser de dos clases: aerobia y anaerobia.

Respiración aerobla

Se realiza en presencia del oxigeno del aire: dando como productos finales gas carbónico, agua y energla calórica.

\section{Respiración anaerobia}

Se hace en ausencia del oxigeno del aire: da como productos finales en los animales ácido acético y energía y en los hongos gas carbónico, alcohol y energia.

\section{Respiración en células}

El proceso respiratorio de todos los seres vivos se realiza a nivel celular; las sustancias protoplasmáticas se oxidan liberando energía gracias a la acción de las enzimas producidas por las mitocondrias.

Mitocondria: tiene comø función producir enzimas que ayudan a la función respiratoria.

Parte de la energía liberada de las sustancias protoplasmáticas (glucosa), se pierde en forma de calor, parte se utiliza en la misma célula y el resto se almacena en las moléculas almacenadoras y transportadoras de la energía ADP y ATP (Adenosín Difosfato, Adenosin Trifosfato).

\section{Respiración en moneras}

Algunas bacterias realizan fotosintesis, es decir producen ATP a partir de la energía solar. Este ATP es utilizado para producir alimentos. Entre las bacterias no fotosintetizadoras, algunas presentan respiración anaerobia y obtienen la energia por procesos de fermentación sin necesitar el oxigeno atmosférico.

La mirocondria es llamada la "central energética de la cétula".
Otras bacterias presentan respiración aerobia y oxidan sustancias organicas mediante el oxigeno. Finalmente, unas cuantas bacterias son facultativas, es decir, pueden presentar respiración aerohia o anaprohia dependiendo del oxigene disponible en el medio. 
Estandar de contenido; Conazco las clases de respiracion.

\section{Respiración en protistos}

Protistos como la ameba, la euglena y el paramecio tienen respiración aerobia. Ellos toman el oxigeno disuelto en el agua o en Ios liquidos del hospedero y liberan el gas carbonico gracias a mecanismos de difusión

Los de vida parásita, como \&̊flasmodio, tienen también respiración aerobia; el intereambio gaseoso lo realizan con los liquidos del hospedero: toman el oxigeno y le ceden el gas carbónico por difusión.

\section{Respiración en hongos}

Los hongos presentan respiración anaerobia.

Fermentación: este tipo de respiración da como productos finales gas carbónico, alcohol y energía. En el ámbito industrial esta característica de los hongos es aprovechada para la fabricación de licores como vinos y cervezas.

Productos realizados a partir de procesos de fermentación.

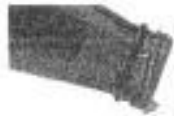

\section{Respiración en plantas}

Las plantas necesitan energla para cumplir sus funciones vitales; esa cnergin la suministra lu rcspiración. Intercambio gaseoso: las plantas inferiores y algunas acuáticas lo hacen por difusión; las superiores poseen organos especializados localizados en diferentes partes de la planta. Éstos son: estomas, lenticelas y neumatóforos.

Estomas: son los órganos más importantes para el intercambio gaseoso; se localizan en el envés de las hojas y en los tallos verdes.

Están formados por 2 células arriñonadas llamadas oclusivas, que dejan entre ellas un orificio llamado ostiolo; éste se abre y se cierra permitiendo la comunicación del medio con una cavidad llamada estomática, la cual se comunica con los espacios intercelulares del parénquima por donde se difunden los gases hasta las células.

Las lenticelas: son aberturas ovales de los tallos adultos; cumplen con la misma función que los estomas.
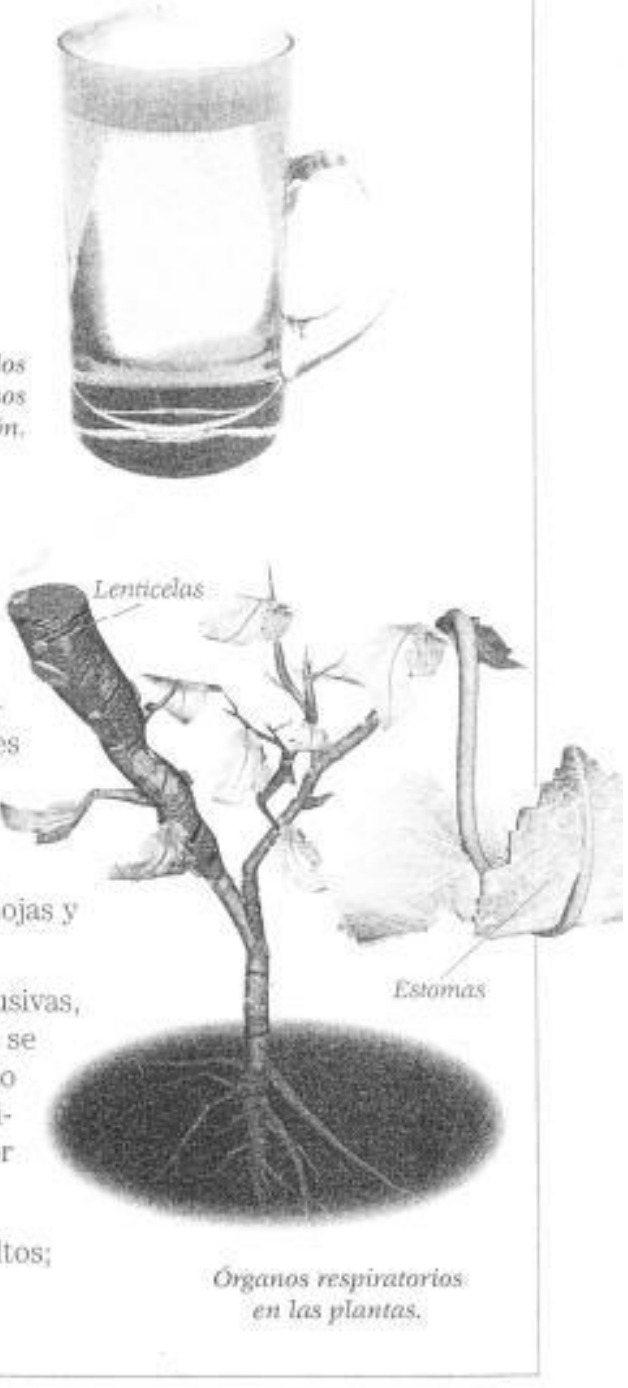
Los neumatóforos: son especializaninnes en forma de tubo de las raices de plantas de pantano; por ellos toman el aire.

\section{Respiración en animales}

La liberación de la energia en los af̧ 7 ales se realiza en las celulas. Los animales tienen respiración aerobia.

Intercambio de los yases. La incorpuración del vxigeno y la eliminación del gas carbontieo se puede realizar de 5 formas diferentes que originan los 5 tipos de respiración.

Tipo de
respiración

Respiración
dirmetia

Resyiración traqueat

Respisación branquial

Fespiración
putmonar

\section{Caracteristicas}

Cuardo el intercambio de gases se realiza entre el medio y las celulas a traves de la mem brana citoplasmática por el fenrímeno de difu. sion; te oxigeno combuste las sustancias protoplasmáticas y libera la energia.

Consiste en la difusión del oxigeno a través de la prel o legumento hasta las vasos sanguineos y la eliminación del gas carberrico desde los vasus sanguínxos por la piet al medio. La sangre transpona cl oxigenn y a nivel celular realza un nuevo intercambin de gases respiratorios por difusión: el oxigeno combuste las sus tancias protoplasmáticas y libera la energia.

Cuando el intercambio gaseoso se realiza por medio de órganos espocializadios llamados tráqueas (o tilotráqueas)

\section{Mecanismo respiratorio:}

medio $\rightarrow$ tráquea $\rightarrow$ células $\rightarrow$ tráquea $\rightarrow$ medio

Cuando el intercambio gaseoso se realiza por medio de branquias; es propia de animales acubiticos.

\section{Mccanismo respiratorio:}

ağua $\rightarrow$ branquia $\rightarrow$ sangre $\rightarrow$ célula $\rightarrow$

sangre $\rightarrow$ branquia $\rightarrow$ agua.

Cuando el intercambio gaseoso se hace en organos especializados llamaklos pulmones que bacen parte del aparato respieatorio.

\section{Organismos que \\ la realizan}

Poriferon, celenterados. platelmintos iexcluyendo la planaria) y nemátodos.

Platelmintos (planaria). anélidos, algunos moluscos (bathosal) y anfibios.

Artrópodos de vida terestre: atacnidos, insectos. quilópodos iciempiési y diplópoctos imilpiesi.

Equinodermos yestrella. enzo de man: crustáceos icangrejo, langosiat: mo tuscos fostras y calama res) y peces.

Anfibios, reptiles, aves. peces pulmonados. 


\section{Apéndice 2}

Estrindar de contenido: Describo el comportamiento y les caracteristicas de los fuidas.

\section{Los fluidos}

A los cuerpos que tienen la propiedad de fluir como los líquiđos y los gases se les denomina flutdos. Éstos adaptan su forma a la del recipiente que los contiene ya que las moléculas que los forman están en continuo movimiento.

\section{Propiedades de los líquidos}

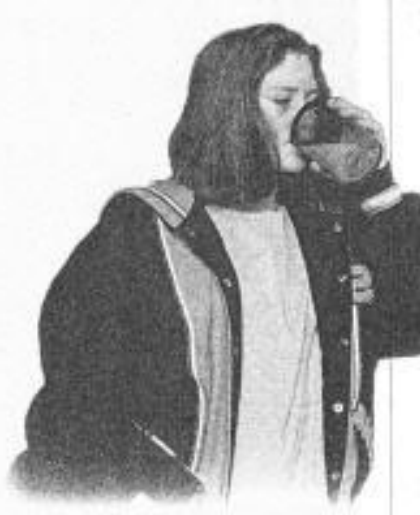

Las liquidos adquieren la forma del recipisnte gue los contiene.

Dentro de las propiedades de los líquidos se destacan:

La forma: los liquidos adquieren la forma del recipiente que los contiene. Esto es debido a que la fuerza de cohesión entre las moléculas del agua es muy débil lo cual permite separar las particulas,

Los liquidos son incompresibles, es decir su volumen no disminuye al ejercerles fuerzas muy grandes. Esto se debe a que las moléculas de agua se encuentran a una distancia tal que no es posible acercarlas más.

La densidad: las diferentes sustancias que existen en la naturaleza se caracterizan porque para un mismo volumen tienen diferente masa. Asi por ejemplo, la masa de 1 centimetro cúbico de cobre es 8,9 gramos, mientras que el mismo volumen de alcohol tiene una masa de 0,81 gramos.

La densidad de una sustancia es la masa por la unidad de volumen de dicha sustancia.

Si una masa m ocupa un volumen $v$, la densidad d és igual a: $\mathrm{d}=\mathrm{m} / \mathrm{v}$

La presión: el peso del libro que actúa sobre la superficie de menor área ejerce mayor presión. La presión depende de dos factores: la fuerza aplicada y la superficie sobre la que actua.

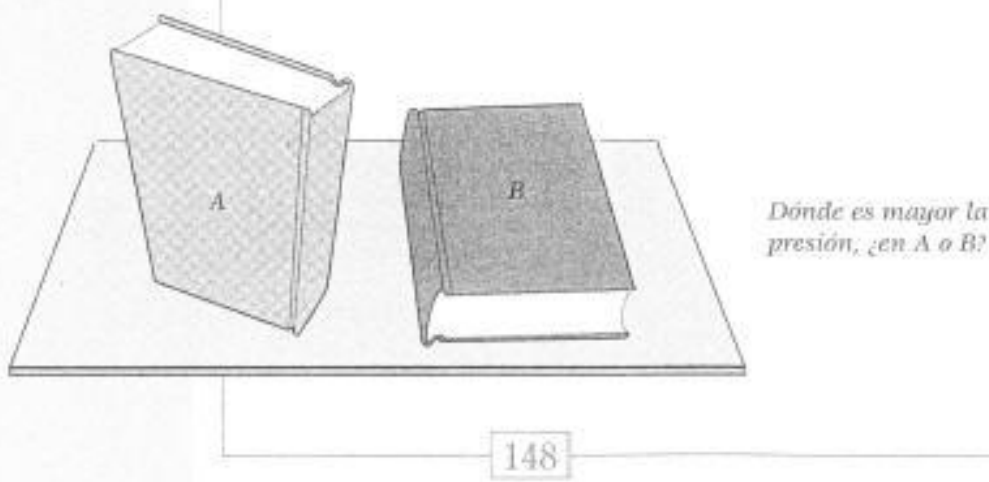

